\title{
Review of: "Variations in tissue optical parameters with the incident power of an infrared laser"
}

\author{
Guennadi Saiko ${ }^{1}$ \\ 1 Ryerson Polytechnic University
}

Potential competing interests: The author(s) declared that no potential competing interests exist.

The manuscript "Variations in tissue optical parameters with the incident power of an infrared laser" by Hamdy and Mohammed is dedicated to investigating the dependence of the optical parameters (absorption and reduced scattering) of turbid tissue on the illumination intensity.

While the research idea is generally interesting for therapeutic applications like LLLT, the methods are not adequately designed and described.

In particular, I have a significant issue with this article. The primary effect of the light-tissue interaction in this power range will be heating the sample, which may result in multiple effects, starting from protein denaturation to water evaporation, coagulation, etc. Thus, several questions arise:

1. The beam's cross section and the power density are more important than the light power. This is because they will affect the temperature distribution in the samples. However, they were not reported.

2. The data were collected in vitro; thus, the realistic heat dissipation mechanisms (like blood flow) are absent, and tissue water content is different. Therefore, it may result in an entirely inadequate (overestimated) temperature distribution, which does not have relevance to in vivo.

3. The duration of the treatment is also important for the temperature distribution. Is it steady-state or pulsed? The tissue heating will be a transient process. It is not clear at what point of this process data were captured

Thus, it would be more important to do thermal simulations than optical simulations in this case. With these omissions in the experimental design, I don't see any practical use of these results. 